AUSENCIA DE UNA NORMATIVA CONTABLE Y FISCAL COMO BASES DE REVISIÓN EN MATERIA AMBIENTAL EN MÉXICO

\title{
AUSENCIA DE UNA NORMATIVA CONTABLE Y FISCAL COMO BASES DE REVISIÓN EN MATERIA AMBIENTAL EN MÉXICO
}

\section{ABSENCE OF A FINANCIAL REGULATION AND STOCKHOLDERS AS BASES IN ENVIRONMENTAL REVIEW IN MEXICO}

\author{
Murrieta Martínez Natalia*, Ricárdez Jiménez Jerónimo D.**

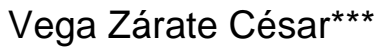

*Licenciada en Contaduría Pública. Maestra en Contabilidad, Área Impuestos. Catedrática de la Universidad Veracruzana.

**Doctor en Ciencias Económicas. Catedrático de la Universidad Veracruzana. jeronimoricardez@hotmail.com

***Licenciado en Contaduría Pública. Maestro en Impuestos y Maestro en Ciencias y Gestión. Doctor en Ciencias Administrativas y Gestión para el Desarrollo. Docente de la Universidad Veracruzana, México. cevega@uv.mx

Dirección para recibir correspondencia: nmurrieta@uv.mx 
AUSENCIA DE UNA NORMATIVA CONTABLE Y FISCAL COMO BASES DE REVISIÓN EN MATERIA AMBIENTAL EN MÉXICO

\section{RESUMEN}

La aparición del término desarrollo sustentable ha generado una importante conciencia social sobre la relación existente entre la naturaleza y el ser humano cuya interacción ha sobrepasado el ámbito ecológico. Así, desde su apreciación en el Informe de Bruntland en 1987, la presencia de la disciplina económica, se muestra como una corriente imperante para lograr que ese proceso de concientización ecológica sea incorporado por las empresas para proteger al medio ambiente.

A partir de esto, conceptos como contabilidad ecológica y fiscalidad verde aparecen como instrumentos necesarios para que las empresas guíen sus acciones hacia un beneficio ecológico a la par de sus fines empresariales. Así, la incorporación de tales fenómenos ha requerido que sus estructuras normativas sean adaptadas para tal fin, buscándose maneras para contaminar menos y ayudar al planeta. Sin embargo, en México ambos conceptos no han evolucionado del mismo modo, limitándose a escasas regulaciones contables y fiscales que no han sido de gran peso para las empresas mexicanas en virtud de que aún lo ven como ajeno a su realidad empresarial.

Basándose en la comparación de la normativa de ambos conceptos con el Indicador Verde de la Bolsa Mexicana de Valores, se detecta que la intención de las empresas en cuidar al planeta va en aumento a pesar de la escasa regulación contable y fiscal. Por lo tanto, se sugiere que la auditoría ambiental formalice su procedimiento de revisión en materia financiera y no se limite al aspecto administrativo y operativo que actualmente se aplica en nuestro país.

PALABRAS CLAVE: Ausencia. Normatividad. Contable. Fiscal. Ambiental. 
AUSENCIA DE UNA NORMATIVA CONTABLE Y FISCAL COMO BASES DE REVISIÓN EN MATERIA AMBIENTAL EN MÉXICO

\section{SUMMARY}

The emergence of the term sustainable development has generated significant social awareness of the relationship between nature and human beings whose interaction has surpassed the ecological aspect. Thus, since its appreciation in the Bruntland Report in 1987, the presence of economic discipline is shown as an imperative current to ensure that the process of ecological awareness is incorporated by companies to protect the environment.

From this, concepts such as green accounting and green taxation appear as necessary tools for companies to guide their actions towards ecological benefit alongside their business purposes. Thus, the incorporation of such phenomena has required its regulatory structures are adapted for this purpose, seeking ways to pollute less and help the planet. However, in Mexico two concepts have not evolved in the same way, limited to few accounting and tax regulations that have not been of great help for Mexican companies due to they do not consider it as their business reality.

Based on the comparison of the rules of both concepts with the Green Indicator in the Mexican Stock Exchange Market, it is detected that the intention of companies in caring for the planet is increasing despite the limited accounting and tax regulation. Therefore, it is suggested that environmental audit must formalize its procedures in terms of financial matters and not only in the administrative and operational aspects which are currently applied in our country.

KEYWORDS: Absence. Normativity. Accountant. Fiscal. Environmental.

\section{INTRODUCCIÓN}

\section{El fenómeno de la sustentabilidad ambiental}

Uno de los aspectos fundamentales implicados en la crítica al modelo de desarrollo en la modernidad industrial actual lo ha sido la temática del medio ambiente como parte de una necesidad que existe en todos los seres humanos en cuidar y proteger a nuestro planeta a través de diversas acciones a favor de la no contaminación. Así, como parte de un proceso de concientización internacional en un sentido ecológico, el tópico de sustentabilidad ha vuelto a resurgir buscándose nuevas maneras de insertarse en el actuar diario de todos los individuos. 
AUSENCIA DE UNA NORMATIVA CONTABLE Y FISCAL COMO BASES DE REVISIÓN EN MATERIA AMBIENTAL EN MÉXICO

Al mismo tiempo que la globalización toma auge en diversos ámbitos, la nueva idea del desarrollo económico lleva implícita una dimensión de carácter ecológico o verde, en donde todos los individuos deberán ejercer prácticas favorecedoras a la estabilidad y cuidado del medio ambiente para crear un beneficio tanto en la salud y calidad de vida de todos.

Según Al Gore, citado por Mires (2009), quien fuera vicepresidente de los Estado Unidos, país que no necesariamente es definido como ecológico, menciona que la época donde el término "ecología" era únicamente reconocido y estudiado por los biólogos, ha quedado en el pasado, marcando así un legado que permite hacer conciencia sobre las relaciones entre la naturaleza y el espíritu humano.

Desafortunadamente, hoy en día se está viviendo una crisis ecológica, manifestada en total medida por las condiciones ambientales y como resultado de una falsa percepción del rendimiento de los recursos naturales. Por lo tanto, es necesaria la creación de modelos y propuestas que permitan un crecimiento bajo los términos de la sustentabilidad, lo que permitirá visualizar diferentes posturas ante dicha situación.

El concepto de sustentabilidad emerge del reconocimiento de la función que cumple la naturaleza como soporte, condición y potencial del proceso de producción (Leff, 2002), en donde desafortunadamente una de las características en el modelo de desarrollo tradicional basado en el crecimiento económico es que se "desterró a la naturaleza de la esfera de la producción, generando procesos de destrucción ecológica y degradación ambiental...La crisis ambiental vino a cuestionar la racionalidad y los paradigmas teóricos que han impulsado $y$ legitimado el crecimiento económico, negando a la naturaleza. La sustentabilidad ecológica aparece así como un criterio normativo para la reconstrucción del orden económico, como una condición para la sobrevivencia humana y un soporte para logar un desarrollo durable, problematizando las bases mismas de la producción" (Leff, 2002).

Ante tales situaciones, empiezan a surgir a nivel internacional movimientos con el fin de minimizar el impacto que estas amenazas estaban generando. Por mencionar los más representativos está el Club de Roma en 1968, las recomendaciones y estrategias por parte de la Comisión Económica para América Latina y el Caribe (CEPAL) en el 2002, así como la Asamblea General de las Naciones Unidas (AG), en la Conferencia de las Naciones Unidas sobre el Medio Humano en Estocolmo en 1972 a partir de la aprobación de diversas disposiciones financieras e institucionales para la cooperación internacional en materia ambiental. 
AUSENCIA DE UNA NORMATIVA CONTABLE Y FISCAL COMO BASES DE REVISIÓN EN MATERIA AMBIENTAL EN MÉXICO

No obstante, el documento principal reconocido como impulsor de la conciencia ecológica en un sentido económico se dio en el año de 1987 a través del documento denominado "Nuestro futuro en común" mejor conocido como el Informe de Bruntland, con el fin de mostrar la necesidad de dirigir un desarrollo sostenible, considerándose como un concepto de carácter permanente en los discursos del Cooperativismo Internacional (ONU, 2012). En el documento se establece la necesidad de diseñar modelos de producción y consumo que den respuesta a la problemática señalada, surgiendo esta iniciativa como parte de dicho informe, donde se define al desarrollo sustentable como: "El desarrollo que satisface las necesidades de la generación presente sin comprometer la capacidad de las generaciones futuras para satisfacer sus necesidades" (ONU, 2012).

Aunado a lo anterior, la ONU considera prudente lograr:

- Un crecimiento económico con equidad en donde se formalice un sistema económico mundial con el atributo de responsable, en donde ninguna nación o comunidad quede rezagada.

- Una conservación de recursos naturales y del medio ambiente, en donde a partir de la elaboración de soluciones económicamente viables se reduzca el consumo de recursos naturales, se detenga la contaminación y se conserven los hábitats naturales, con el fin de preservar el patrimonio ambiental y recursos naturales para las generaciones futuras.

- Alcanzar un desarrollo social, en donde se atiendan las necesidades de empleo, alimentación, energía, atención a la salud, abastecimiento de agua y saneamiento. Lo anterior, implica un respeto a la diversidad cultural y social.

Las intenciones anteriores se han ido fortificando a partir de diversas actividades mundiales, tales como la denominada "Cumbre para la Tierra" celebrada en 1992 como un plan de acción que permita implementar medidas que ayuden a lograr un desarrollo sustentable. De igual modo, en septiembre de 2000, en la Cumbre del Milenio de las Naciones Unidas, se diseñaron objetivos que permitieran combatir los principales desafíos del mundo denominados como "Objetivos de Desarrollo del Milenio" siendo uno de ellos el relacionado con la temática ambiental (Banco Mundial). Dos años después se lleva a cabo la "Cumbre de Johannesburgo cuyos objetivos son el seguimiento de los compromisos acordados en reuniones anteriores. 
AUSENCIA DE UNA NORMATIVA CONTABLE Y FISCAL COMO BASES DE REVISIÓN EN MATERIA AMBIENTAL EN MÉXICO

Como puede observarse, la temática del medio ambiente ha sido objeto de análisis en diferentes foros internacionales, cuyo factor en común es la preocupación por cuidar el planeta, buscándose nuevas formas por contrarrestar los daños causados por la acción humana.

Es entonces que a partir de tal preocupación, diversas áreas se han interesado en insertar en su contexto de análisis la dimensión ecológica para coadyuvar con las finalidades diversas y alcanzarse un desarrollo sostenible. Dentro de esto, la idea de ligar dicho desarrollo con aquel de tipo económico ha sido una pieza fundamental en donde las actividades económicas se vuelven punto de interés para disminuir los efectos contaminantes. Así, la aparición de la economía verde o sustentable denota un grado de importancia de la cual se derivan otras disciplinas en su entorno encargadas de controlar y vigilar sus recursos no solamente en su dimensión económica, sino también ecológica. Tal es el caso de la contabilidad verde o ecológica y la fiscalidad verde, considerados como fenómenos para las empresas que surgen a partir de loa intención del desarrollo sostenible expuesto anteriormente.

\section{La contabilidad ecológica o verde}

El hacer mención del término contabilidad para toda empresa es referirse a una técnica indispensable que es utilizada por las empresas para fines de control y generación de información de carácter financiera para facilitar la toma de decisiones. Dicha disciplina es de carácter social en virtud de que emerge de las necesidades del hombre por organizarse y por consumir bienes materiales y no materiales que deben ser controlados de manera monetaria. Actualmente, estas necesidades han sido satisfechas por las organizaciones, visto desde la teoría de la agencia y la teoría general de la organización, en la que el hombre busca la reducción de costos para hacerse llegar de productos y servicios que le faciliten la operación de su organización o su persona; en algunos momentos esto se logra sobre el uso irracional de los recursos que nos proporciona la naturaleza y el registro de estas transacciones, que deben convertirse en monetarias. Lo anterior, es el objeto de la contabilidad.

Al término de contabilidad se le añade de manera común apellidos como: Contabilidad Financiera, aquella que se encarga de controlar el aspecto de planeación financiera de la empresa, el control de su estructura financiera (activos, pasivos y capital); Contabilidad Fiscal, aquella que además de convertir monetariamente las transacciones económicas de la entidad busca la obtención de información que utilice la empresa para tomar decisiones de tipo tributario; Contabilidad Administrativa, la que se ocupa de organizar la empresa, tomando 
AUSENCIA DE UNA NORMATIVA CONTABLE Y FISCAL COMO BASES DE REVISIÓN EN MATERIA AMBIENTAL EN MÉXICO

decisiones a partir de análisis de costos de operación y producción, además de logística general de la empresa.

Sin embargo, a partir de la aparición de la problemática planteada del cambio climático y el aumento de consumo de recursos como consecuencia del hombre sobre los recursos naturales, esta disciplina incorpora en su estructura esa misma preocupación surgiendo una nueva rama denominada Contabilidad Ambiental, Verde o Ecológica. Conforme a Mantilla (2006), la contabilidad ambiental es:

"El sistema que permite el reconocimiento, organización, valoración y registro de las condiciones y cambios en los recursos naturales y del ambiente, articulando indicadores de evaluación de la sostenibilidad ambiental en el contexto de desarrollo, estableciendo sistemas de información que faciliten el control y fiscalización de las acciones que afectan la condición de la naturaleza y el desarrollo nacional".

Se ha considerado incluir otro nuevo "tipo" de contabilidad que haga frente al aspecto de la utilización en la empresa de recursos naturales, tanto renovables como no renovables en su sentido verde o ambiental o ecológico, empleado como sinónimos hoy en día (Pérez, 2009). Contablemente, los efectos medioambientales que sean causados por la empresa, pueden considerarse como una pérdida, por ejemplo, lo relacionado con los gastos de daño ambiental (impuestos por autoridades ambientales, como es el caso de México), que paguen las empresas que "ocasionen el daño", representan de acuerdo a las NIF una disminución de sus activos o incremento de sus pasivos, otro ejemplo son las pólizas de seguros que cubren daños ambientales, las cuales tienen vigencia de un año (corto plazo), sería conveniente clasificar su pago como un activo amortizable, que con el tiempo y su devengación contable se convierta en gasto.

El indicio de la expresión ambiental, ecológico o verde, como tópico importante para las ciencias sociales surge con la llamada "economía ambiental" término que de acuerdo con Pichs (2011) aboga por la sostenibilidad basada en la aplicación del principio de que "el que contamina paga", a partir de la aplicación preferente de los mecanismos de mercado (impuestos ambientales, permisos de emisión negociables). Según este enfoque, es posible contar con métodos económicos de mercado que permiten obtener, de forma eficiente, un nivel óptimo de degradación ambiental y una valoración económica del daño ambiental, como base para la toma de decisiones. 
AUSENCIA DE UNA NORMATIVA CONTABLE Y FISCAL COMO BASES DE REVISIÓN EN MATERIA AMBIENTAL EN MÉXICO

En este sentido, el término Contabilidad Verde es entendido como el campo de conocimiento que estudia sistemas ambientales y naturales, altamente complejos, en su relación con la actividad económica desarrollada por las sociedades. Desde luego, su estructura teórica se referencia desde un tipo de racionalidad ambiental, que resulta determinante a la hora de utilizar el hábitat y los recursos naturales, en general, estudia las correspondientes unidades ambientales como totalidades en sus aspectos estáticos como dinámicos, su estructura de relaciones, sus correspondientes impactos y procesos de interacciones bióticas y abióticas (Barraza y Gómez, 2005).

Es por ello, que la concepción de este tipo de contabilidad, propone la representación de las relaciones de desarrollo con el medio ambiente y se configure como algo útil para el diseño de las políticas gubernamentales de tipo económico y ambiental, como menciona Barraza y Gómez (2005), la contabilidad a través de la información que presenta, fundamenta decisiones de alta influencia en los procesos de desarrollo enmarcados en el orden de sostenibilidad, con lo que los sistemas de cuentas nacionales pasan de poseer un discreto desempeño en las funciones del Estado, a convertirse en una herramienta por medio de la cual el país se inserta en la nueva dinámica económica.

\section{La fiscalidad ecológica o verde}

Siguiendo el tenor de la contabilidad ecológica, la aparición de nuevas formas de proteger al medio ambiente a través de instrumentos artificiales construidos por el propio hombre en defensa del medio ambiente es latente. Así, dicha concientización hacia el planeta se ve dimensionada en un ámbito en particular del Estado, en donde las acciones por proteger al planeta son tomados en consideración por el poder tributario del Estado, en donde es través de la recaudación de los tributos que pagan aquellas empresas o personas con carácter de contaminante, en donde de algún modo se obliga a reducir los problemas ambientales ya explicados.

Partiendo de la noción de la actividad financiera de todo Estado, cuya preocupación esencial es la de crear satisfactores sociales para su colectividad, reflejadas en servicios públicos, infraestructura social y otras maneras de generar una calidad de vida, el sentido de protección al medio ambiente es incorporado por el Estado a través de la definición de lo que se le conoce como fiscalidad ecológica o verde. 
AUSENCIA DE UNA NORMATIVA CONTABLE Y FISCAL COMO BASES DE REVISIÓN EN MATERIA AMBIENTAL EN MÉXICO

"Conceptualmente se entiende por fiscalidad al conjunto de leyes, reglamentos procedimientos relativos a las tasas, impuestos y contribuciones especiales. La fiscalidad ambiental debe perseguir el cambio de actitudes de los agentes económicos para el medio ambiente. Los impuestos ambientales reducen la contaminación y tienen beneficios económicos porque disminuyen los gastos" (Roccaro y Fernández, 2012).

Así, la fiscalidad ecológica comprende la aplicación de instrumentos económicos de carácter fiscal (recaudatorios) y parafiscales (incentivadores) con el fin principal de propiciar cambios de conducta en los agentes económicos favorables a la sustentabilidad, por encima de los fines estrictamente recaudatorios. (Sandoval, 2008) Es entonces que la finalidad de la fiscalidad se ve reducida a lograr un desarrollo sostenible y perdurable por parte de los agentes contaminantes como consecuencia de sus actividades industriales en calidad de contribuyentes (PwC, 2013).

Si bien es cierto, que la actividad financiera de todo Estado tiene una parte de su soporte financiero vía recaudación de los tributos de los contribuyentes en un espacio y territorio determinado, en un sentido ambiental, dicho modus operandis es traducido ahora en una manera de concientizar al ser humano a que si contamina, ahora debe pagar por ello.

El término de la fiscalidad verde o ecológica se encuentra dado en el Informe de Bruntland (1987) como parte de las acciones de la ONU, en donde se detectaron dos grandes problemáticas:

- Problemas medioambientales, tales como el calentamiento global y la destrucción de la capa de ozono por un lado, y

- La ausencia de instituciones multilaterales que pudieran definir medidas o criterios suficientes para contrarrestar los problemas anteriores.

Bajo la idea anterior, los textos y estudios de la ONU parten de un marco básico: el tributo medioambiental pretende afectar el comportamiento de las personas que como consecuencia de sus actividades económicas, industriales principalmente, dañan o perjudican al medio ambiente de algún modo, convirtiéndolos entonces en responsable ecológicos. Para delimitar lo anterior, dicho organismo propone una cierta categorización de instrumentos necesarios para controlar esto, quedando de la siguiente forma: 
AUSENCIA DE UNA NORMATIVA CONTABLE Y FISCAL COMO BASES DE REVISIÓN EN MATERIA AMBIENTAL EN MÉXICO

- Instrumentos legislativos: compuestos por normas y reglamentos que regulan de algún modo los efectos contaminantes.

- Instrumentos económicos: la fiscalidad ambiental, ecológica o verde.

- Instrumentos voluntarios: plasmados en sistemas de gestión medio ambiental, tales como normas ISO y demás que culminan con una certificación de no contaminación o reducción de contaminantes.

Como se puede apreciar, la fiscalidad ambiental para la OCDE es más que nada un instrumento cuya esencia económica se ve dimensionada en acciones particulares para los contribuyentescontaminantes por sus acciones. Es entonces que la fiscalidad verde es el resultado de la interacción de la economía y la ecología, en donde como consecuencia de la ruptura del equilibrio ecológico por la mano del hombre, fue necesaria la creación de un medio coactivo para regular tal situación.

Ante lo anterior, se plantea la pregunta: ¿por qué la economía se ve inmiscuida en esta instrumentación por parte del Estado y se ve expresada en una recaudación tributaria como parte de un proceso de concientización ecológica en los actores contaminantes? Puede considerarse que una primera respuesta podría darse por el hecho de que la acción de tributar por parte de los individuos obedece a un comportamiento económico, el cual es transformado en una capacidad contributiva que se encuentra definida por una legislación tributaria. Es por ello que, siguiendo la esencia de una teoría tributaria, el Estado recurre a esta acción coercitiva precisamente como un instrumento factible de concientizar a través de los impuestos.

Carballo (2012) señala que "los tributos son instrumentos, herramientas para conseguir el nuevo paradigma; pero no son los únicos útiles de trabajo con los que cuentan las Políticas Públicas para modular los comportamientos humanos a favor de una nueva sociedad $y$, asimismo, ni son omnipotentes, ni omniscientes; por ello, deben colaborar a la hora de construir el nuevo paradigma y actuar en armonía con otras intervenciones públicas y privadas."

En ese sentido, este tipo de fiscalidad se encuentra estructurada por tres grandes ejes:

a) Impuestos, en donde la creación de las denominadas "ecotasas" son gravámenes particulares sobre aquellos sujetos que como consecuencia de un alto índice de contaminación se ven obligadas a pagar una cierta cantidad. En sí, este impuesto es un pago obligatorio por parte de un agente relacionado con la descarga de sustancias contaminantes cuyo objetivo es limitar el deterioro ambiental. 
AUSENCIA DE UNA NORMATIVA CONTABLE Y FISCAL COMO BASES DE REVISIÓN EN MATERIA AMBIENTAL EN MÉXICO

En este concepto, los impuestos pueden ser clasificados en función de las emisiones y de los productos realizados por la industria. Astudillo (2008) nos ofrece la siguiente delimitación de impuestos ambientales en cuanto su estructura:

\begin{tabular}{|c|l|}
\hline Sujeto Pasivo & $\begin{array}{l}\text { Sería tanto el productor como el consumidor de productos } \\
\text { contaminantes y el titular de los bienes patrimoniales con los que se } \\
\text { realiza la actividad contaminante. }\end{array}$ \\
\hline Base Imponible & $\begin{array}{l}\text { Es la medida del daño que se pretende evitar, se ha dicho que en } \\
\text { este caso el impuesto no debe ser con relación a la riqueza sino en } \\
\text { función de la contaminación. }\end{array}$ \\
\hline Tasa del impuesto & $\begin{array}{l}\text { Puede establecerse por la prestación de servicios ambientales, en } \\
\text { este caso suele diferenciarse el costo del servicio de acuerdo con } \\
\text { las conductas más o menos contaminantes. El otro tipo de tasa es } \\
\text { por el aprovechamiento especial de los bienes ambientales que son } \\
\text { de dominio público. }\end{array}$ \\
\hline
\end{tabular}

Figura 1. Elementos del impuesto ecológico.

Fuente: Elaboración propia.

b) Depósitos reembolsables, y

c) Permisos de derechos de emisión. En este punto, debemos forzosamente hacer referencia al Régimen de Comercio de Derechos de Emisión de la Unión Europea, consistente en fijar un "límite máximo" a las emisiones totales de las instalaciones reglamentadas, como las centrales eléctricas. Dentro de este límite, las instalaciones reciben y compran derechos de emisión de gases de efecto invernadero hasta un determinado tonelaje cada año. Si emiten menos, pueden vender los derechos sobrantes. Si prevén emitir más de lo que les permiten sus derechos, pueden elegir entre invertir en medidas o tecnologías de reducción de las emisiones o comprar derechos adicionales de emisión en el mercado que cubran en todo o en parte ese exceso. Esta posibilidad de comerciar, dentro del límite máximo global de las emisiones, crea flexibilidad. Consigue que las emisiones se reduzcan allí donde resulta más barato y orienta las inversiones hacia donde pueden conseguir el mayor ahorro de emisiones (Comisión Europea, 2014). 
AUSENCIA DE UNA NORMATIVA CONTABLE Y FISCAL COMO BASES DE REVISIÓN EN MATERIA AMBIENTAL EN MÉXICO

Específicamente sobre los impuestos ambientales, el definirlos es algo complejo por dos motivos (Carballo, 2012), la primera porque se concreta en una diversidad instrumental que produce bastante confusión y en segundo lugar, porque no se fundamenta en un criterio común que sea relevantes. De algún modo, la operatividad de este tipo de fiscalidad se muestra como un fenómeno complejo en donde la delimitación de los elementos básicos de los impuestos (sujeto, objeto, base, tasa y/o tarifa) persigue fines no fiscales en primera instancia, sino parafiscales, caso contrario a un impuesto no ecológico.

Respecto a la operatividad y efectividad de la fiscalidad verde, una problemática es latente en el sentido de la carga fiscal, de los efectos distributivos de los ingresos fiscales recaudados por ese impuesto ecológico y la efectividad que se está teniendo de ellos. Para ello, será necesario el conocer de qué manera cada país interesado en contrarrestar los efectos contaminantes asume sus políticas públicas ambientales y respetan su legislación tributaria verde.

El alcance de la fiscalidad verde está evidenciada en el año de 1990 en la Comunidad Europea, misma que hasta la fecha es la que ha desarrollado y puesto en prácticas más acciones integradoras de protección al medio ambiente a través del desarrollo de acciones tributarias derivada de una política ambiental preocupada a definir estrategias y acciones de control ambiental. El mayor ejemplo es la denominada "Estrategia comunitaria para delimitar las emisiones del CO2 y mejorar la eficiencia energética", cuyo resultado fue el crear el Impuesto europeo sobre el carbono.

La tributación en este sentido, nace como un instrumento para reorientar las estructuras productivas y las pautas de consumo hacia una menor intensidad carbónica y energética. Con el paso del tiempo, en la Comunidad Europea hoy en día se contempla el Derecho de emisión, el cual de algún modo ha servido para contrarrestar el daño al medio ambiente. De algún modo, la recaudación de ingresos a partir de la fiscalidad verde forma parte de la estructura del PIB de los países europeos, en donde su importancia por definir una estabilidad en este rubro ha sido objeto de estudio y discusión en el Parlamento Europeo. 

AMBIENTAL EN MÉXICO

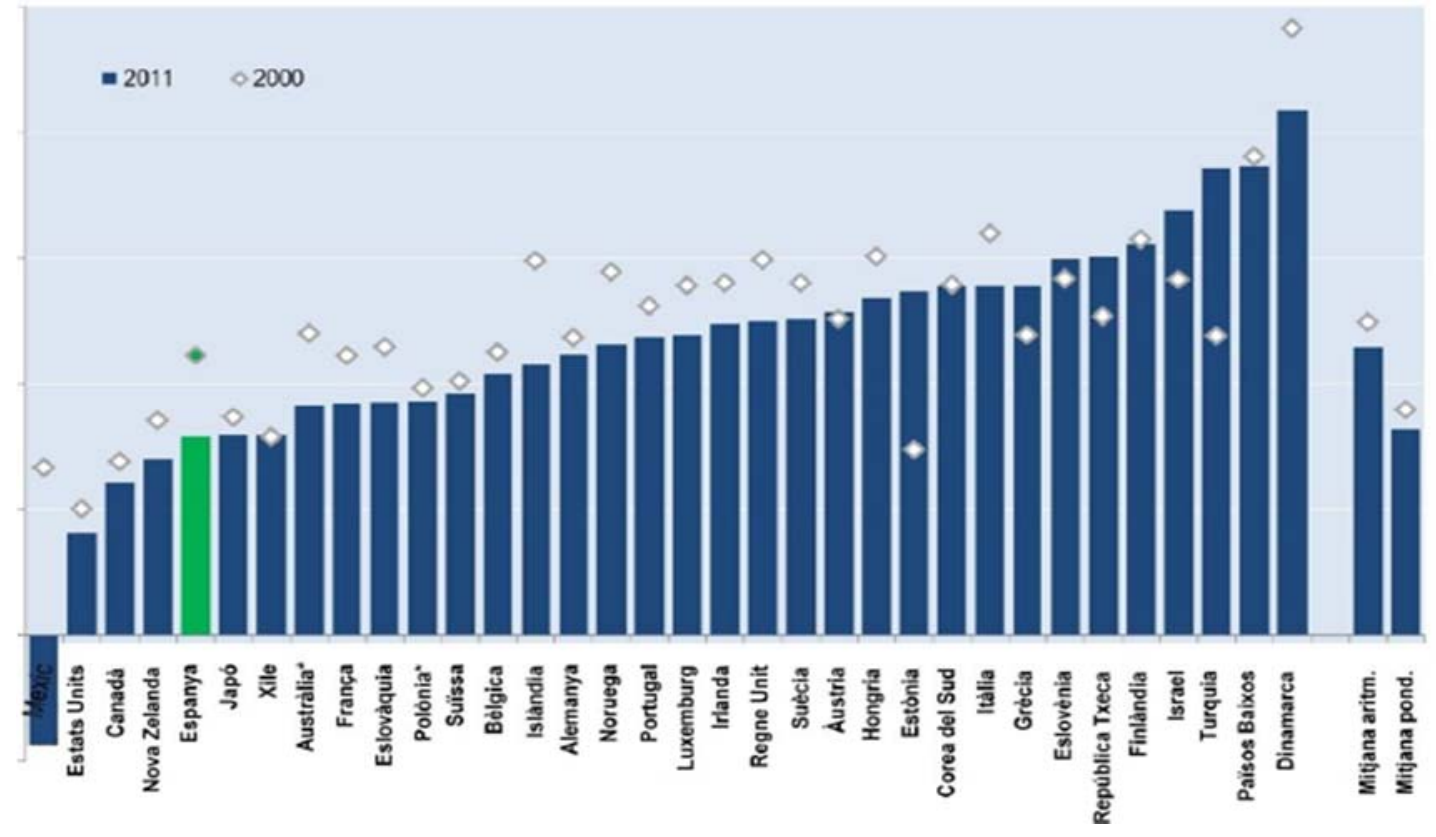

Figura 2.Tendencia recaudatoria de los tributos verdes en Europa, Periodo 2000-2011.

Fuente: Fundación Fórum Ambiental. Fiscalidad ambiental e instrumentos de financiación de la economía verde, 2014.

Como se puede apreciar, el hecho de que Europa esté integrada como una comunidad, ha traído como resultado que la concientización por proteger al planeta sea mucho más recurrente, como parte de sus acciones y políticas públicas ambientales que ha servido como un medio para ayudar nuestro entorno. "La existencia misma de la fiscalidad ambiental significa el reconocimiento de que cuando se presentan impactos ambientales se justifica la participación del Estado para regular o eliminar dichos impactos, aplicando medidas que posibiliten la internacionalización del costo ambiental en la función de costos del agente que realiza la contaminación o hace el uso del servicio o del bien medioambiental" (Sandoval, 2008).

En sí, este tipo de fiscalidad hoy es más que nada una herramienta económica creada con la intención no de ser una fuente de financiamiento directa para el ejercicio de la actividad financiera de un Estado, sino más que todo como un instrumento social consientizador si lo queremos denominar así, el cual promueve a que los contribuyentes contaminantes no lo hagan, o por lo menos reduzcan sus emisiones dañinas para el planeta. Lo anterior, es desde una perspectiva ecológica. Bajo un enfoque económico y jurídico, la fiscalidad verde se convierte como un medio más para recaudar más ingresos para el Estado, cuya imposición depende de las acciones contaminantes de los sujetos pasivos, en donde la capacidad 
AUSENCIA DE UNA NORMATIVA CONTABLE Y FISCAL COMO BASES DE REVISIÓN EN MATERIA AMBIENTAL EN MÉXICO

económica no se convierte en su capacidad contributiva, sino más bien su capacidad contaminante como consecuencia de sus acciones operativas.

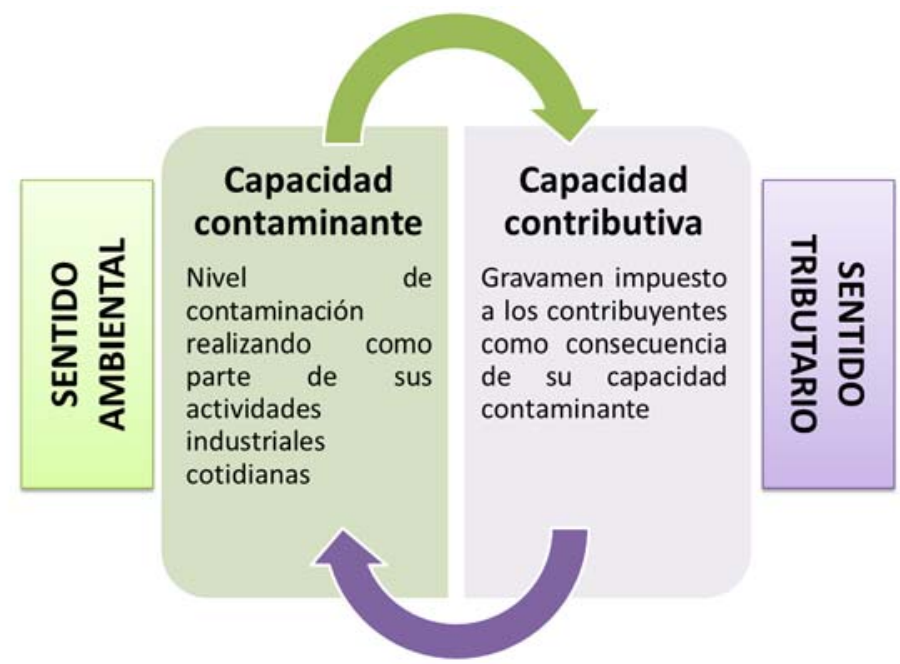

Figura 3. Dimensión de las capacidades ambientales para la fiscalidad verde.

Fuente: Elaboración propia.

\section{Normativa contable y fiscal verde en México}

Como se ha explicado, la presencia de estos dos fenómenos en la gestión de las empresas ha sido importante a nivel internacional, lo cual ha solventado que ambos requieran de una estructura normativa que le permita tanto al Gobierno como a las propias empresas aplicarlos de una manera correcta; es decir, la importancia que tienen ambos conceptos para la reducción de los efectos contaminantes y que salvaguarden los objetivos que se pretenden a nivel internacional. Sin embargo, una pieza fundamental para hacer lo anterior es la parte normativa, que como tarea del Estado, le corresponde definir un marco regulatorio suficiente y competente que permita que las empresas realicen sus actividades económicas a la par con que se cuide al medio ambiente.

En el caso regulatorio de la contabilidad ambiental en México, únicamente existe la siguiente normatividad:

\begin{tabular}{|c|c|}
\hline Norma Mexicana & Regulación específica en materia ecológica \\
\hline Norma C-9: Pasivo, & Específicamente en el reconocimiento de una provisión contable \\
provisiones, activos y & resultante de un evento pasado a cargo de una entidad. Algunos \\
pasivos contingentes y & ejemplos son las multas por reparación por daños al medio ambiente \\
compromisos & o los costos de reparación de éstos; tal incidencia deberá \\
& reconocerse en los estados financieros correspondientes. \\
\hline
\end{tabular}


AUSENCIA DE UNA NORMATIVA CONTABLE Y FISCAL COMO BASES DE REVISIÓN EN MATERIA AMBIENTAL EN MÉXICO

Referente a la fiscalidad ambiental mexicana, a la fecha se puede afirmar que no existe alguna normatividad formal que regule el impacto con el medio ambiente (Sandoval, 2008), en donde únicamente se cuenta hoy en día con una serie de leyes no fiscales preocupadas por el daño ambiental, tales como la Ley Federal de Derechos, Ley General para la Prevención y Gestión Integral de los Recursos, Ley General del Equilibrio Ecológico y la Protección al Medio Ambiente, Ley General del Cambio Climático, entre otros. De otro ángulo, y de manera bastante difusa, el Impuesto Especial sobre Producción y Servicios contempla el pago de un impuesto por la adquisición de plaguicidas de acuerdo a su grado de toxicidad, limitándose al pago de éste a los compradores de dichos productos, situación que está gravada en función de las unidades adquiridas y no por el daño causado, dejando fuera el posible impacto que pudiera tener su consumo para el medio ambiente.

De algún modo, en el contexto mexicano la fiscalidad se está dando a partir de que las prácticas empresariales tendientes a contaminar se vean disminuidas a través de un beneficio fiscal vía estímulo, lo cual obliga a que se busquen nuevos modos para no afectar a la sociedad. Sin embargo, consideramos que esto no lo podemos denominar como fiscalidad ambiental en sí, puesto que de acuerdo a lo abordado en el presente documento, este tipo de fiscalidad cuenta con tipos de atributos que la hacen diferencias de otras medidas de control ambiental, en donde el Estado Mexicano aún no desarrolla instrumentos eficaces de reducción ambiental tal y como lo sugieren los organismos internacionales.

\section{EL IPC verde mexicano}

A pesar de la carencia normativa en materia contable y fiscal en México, es interesante el analizar que a finales de 2011, la Bolsa Mexicana de Valores presentó el Índice de Precios y Cotizaciones Sustentable, el cual se encuentra conformado por los siguientes elementos: medio ambiente, considerando el impacto y riesgos generados conforme al sector en el que opera la emisora en cuestión; responsabilidad social, hacia sus empleados, proveedores, clientes y comunidad en la que realiza operaciones la entidad; y prácticas de gobierno corporativo. (Vásquez, 2014).

Así, anualmente, las emisoras deben hacer público un reporte anual que englobe los programas y acciones desarrolladas en materia sustentable. Al parecer en México las grandes empresas están haciendo sus primeros esfuerzos para convertirlo en acciones que sean reflejadas monetariamente, para la reducción de costos, y que además sean consideradas por la contabilidad para controlar y tomar decisiones financieras. 
AUSENCIA DE UNA NORMATIVA CONTABLE Y FISCAL COMO BASES DE REVISIÓN EN MATERIA AMBIENTAL EN MÉXICO

Tomando como base tal indicador, nos percatamos que el debido comportamiento ambiental ha ido en incremento, lo cual eso sugiere que la preocupación por cuidar al medio ambiente ha sido favorable y que de algún modo las grandes empresas que cotizan en bolsa están cuidando sus prácticas empresariales a favor del medio ambiente.

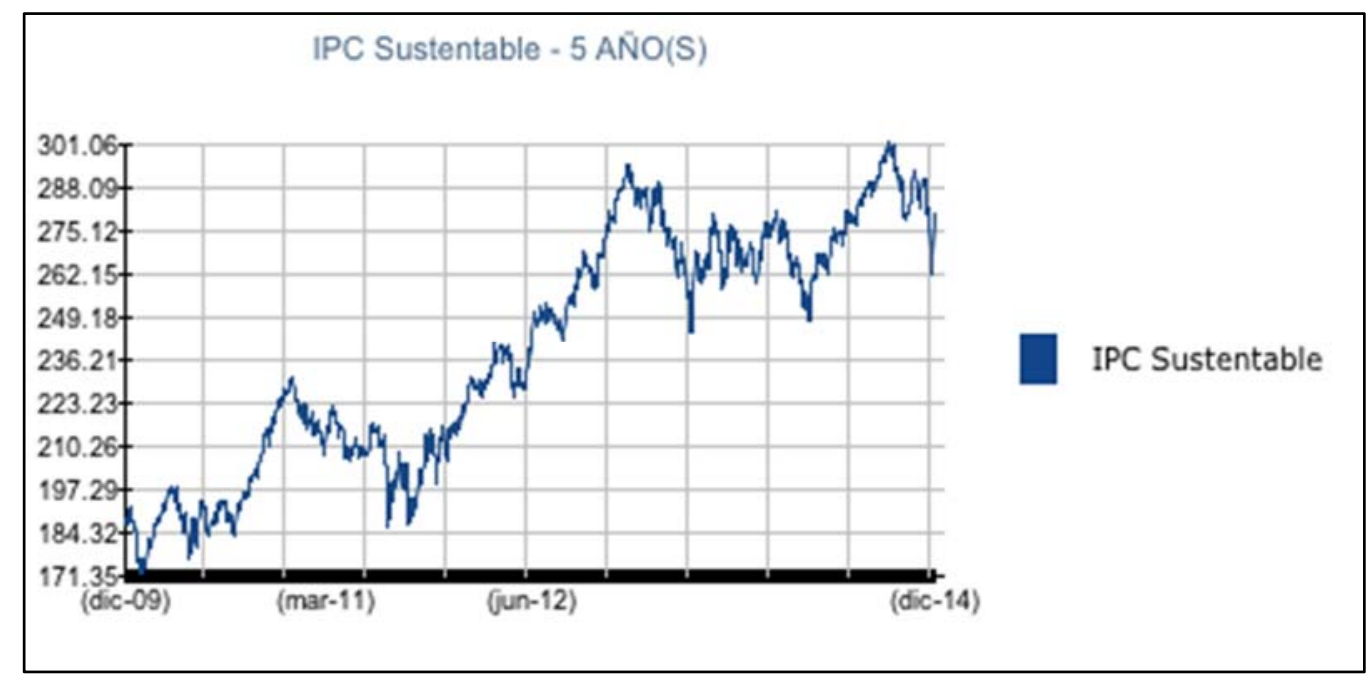

Figura 4. Comportamiento del IPC Sustentable en México.

Fuente: Base de datos de la Bolsa Mexicana de Valores (BMV).

EI IPC Sustentable es de utilidad para las compañías que lo integran porque hay inversionistas, principalmente extranjeros, que buscan apostar por firmas que comprueben su preocupación por el medio ambiente a través de sus prácticas responsables. Tal indicador refleja que únicamente las empresas que cuentan con una fortaleza administrativa y financiera tienden a ser más sustentables a pesar de la carencia normativa. Sin embargo, se detecta que los efectos de disminución únicamente se están dando por la responsabilidad social que grandes empresas asumen, mismas que se convierten en requisitos para permanecer en cotización en bolsa.

\section{Consideraciones finales}

La protección y cuidado al medio ambiente en el ámbito de las empresas poco a poco ha ido formando parte importante en su gestión, en donde debe buscarse nuevos procesos por no dañar al planeta y lograr un equilibrio ecológico y sustentable. Sin embargo, para lograrlo, es necesario que se cuente con una normativa por parte del Estado que se encargue de controlar e imponer las medidas necesarias para ayudar al fin ecológico. En este sentido, particularmente en el caso de México, existe una carencia de normativa por parte del IMCP en materia contable y por la SHCP en materia fiscal, a pesar de tener conocimiento de la importancia del fenómeno ecológico en el ámbito económico y empresarial. 
AUSENCIA DE UNA NORMATIVA CONTABLE Y FISCAL COMO BASES DE REVISIÓN EN MATERIA AMBIENTAL EN MÉXICO

Ante el punto anterior, puede mencionarse que a nivel mundial tanto la contabilidad y fiscalidad ambiental se han ido desarrollando de manera paulatina con la globalización, en donde las acciones no contaminantes no sólo deben dejarse a un ámbito de conciencia social, sino que es necesaria de una normatividad que regule el comportamiento de los agentes económicos y/o contribuyentes de cualquier territorio. Ante este punto, valdría hacerse la siguiente pregunta: si existe una carencia tanto contable como fiscal en México, ¿qué debemos entender por auditoría ambiental?

Actualmente, el evocar el término de auditoría en un sentido ambiental implica únicamente se hacer un check list de las múltiples acciones y requisitos que son necesarias que las empresas tengan para proteger al medio ambiente, lo cual no consideramos como incorrecto, sino que más bien dentro de esta área de revisión no existe participación alguna de la contabilidad financiera que deben estarse realizando por ellas, acompañada de una carencia de normatividad fiscal ecológica. El contar con esas dos dimensiones, el término de auditoría ambiental sería mucho más completo y su inserción en este sistema permitiría un control financiero más efectivo en materia ecológica.

Bajo una perspectiva contable, para que exista la auditoría financiera debe existir una contabilidad que genere información y que sea susceptible de revisión. Lo mismo sucede en el ámbito fiscal, en donde las empresas deberán producir información fiscal basada en su información contable sujeta también a revisión. En sí, eso es lo que hace la auditoría. No obstante, si hacemos un análisis de la auditoría ambiental, definitivamente ésta se encuentra bastante alejada de lo que persigue una auditoría en sí, puesto que ella no sólo se concentra en hacer el check list antes mencionado, sino más bien en dar una opinión, que en materia ambiental sería opinar si la gestión de las empresas son o no contaminantes basadas en otras consideraciones más de fondo.

La reflexión anterior surge por el hecho de saber que en nuestro país, a pesar de una carencia en la normativa contable y fiscal ecológica, ya existe un indicador ambiental basado en un ambiente bursátil y que explica que las empresas sí se están preocupando (al menos las empresas que cotizan en bolsa) en proteger y cuidar al medio ambiente a través de sus prácticas, lo cual sería de bastante interés por parte del gobierno en definir y crear con mucho más precisión un marco normativo que cuide y vigile tal comportamiento empresarial bajo la óptica ambiental. Definitivamente, el hacer lo anterior, haría que tanto la contabilidad, la fiscalidad y la auditoria ambiental estuvieran debidamente conectadas y coordinadas entre sí, 
AUSENCIA DE UNA NORMATIVA CONTABLE Y FISCAL COMO BASES DE REVISIÓN EN MATERIA AMBIENTAL EN MÉXICO

para que en su conjunto las tres disciplinas se incorporen en la gestión empresarial y realmente el fenómeno ambiental estuviese mejor controlado. Se espera que en un futuro las empresas realicen su gestión empresarial con aras a una gestión ambiental efectiva con medidas de contabilización, tributación y revisión adaptadas a su realidad.

\section{REFERENCIAS BIBLIOGRÁFICAS}

Astudillo Moya, M. (2012). La tributación con fines ambientales en México.

Banco Mundial. (2012). Objetivos de desarrollo del milenio. Recuperado de http://www.bancomundial.org

Barraza, E., Gómez, E. (2005). Aproximación a un concepto de contabilidad ambiental. Bogotá, Colombia: Universidad Cooperativa de Colombia, Facultad de Ciencias Administrativas, Económicas y Contables.

Bolsa Mexicana de Valores (2014). www.bmv.org.mx

Carballo Vasco, D. (2012). La economía del cambio climático y la fiscalidad. Crónica Tributaria, pp. 7-44.

Comisión Europea, C. (2013). Acción por el clima. Pp. 1-16.

Fundación Fórum ambiental (2013). Fiscalidad ambiental e instrumentos de financiación de la economía verde, pp. 1-14.

Leff, E. (2012). Saber ambiental, sustentabilidad, racionalidad, complejidad, poder. México, D.F.: Siglo XXI editores.

Mantilla, E. (2006). La contabilidad ambiental en el desarrollo sostenible. Revista internacional de Legis de contabilidad y auditoría, 25, pp. 133-160.

Mires, F. (2009). La revolución que nadie soñó. O la otra posmodernidad. Argentina: Libros de la araucaria.

ONU. (2012). Carta de las Naciones Unidas. Recuperado de www.un.org/es/documents/charter/index.hstml 
AUSENCIA DE UNA NORMATIVA CONTABLE Y FISCAL COMO BASES DE REVISIÓN EN MATERIA AMBIENTAL EN MÉXICO

Pérez, M. (2009). La contabilidad ambiental: una aproximación desde la economía ecológica, adversia. Universidad de Antioquia, 5, Medellín.

Pichs, R. (2011). El debate acerca de la "economía verde". Nueva época UU, 20, La Habana.

PwC. (2013). Fiscalidad verde en Europa. Objetivo 20/20/20.

Roccaro, I., \& Fernández, E. (2012). Fiscalidad medioambiental sobre el sector energético. El caso de Argentina.

Sandoval Cabrera, P. (2008). Análisis comparativo de la fiscalidad ambiental en México y en Europa: opciones de cooperación en el marco del acuerdo de la asociación económica. Revista electrónica Iberoamericana, II, pp. 141-159.

Vásquez Quevedo, N. (2014). Desarrollo de la contabilidad ambiental en México: normatividad, organismos reguladores e indicadores. XIX Congreso Internacional de Contaduría e Informática de la Universidad Autónoma de México. 